\title{
THE AU MIC DEBRIS DISK: FAR-INFRARED AND SUBMILLIMETER RESOLVED IMAGING
}

\author{
Brenda C. Matthews ${ }^{1,2}$, Grant Kennedy ${ }^{3}$, Bruce Sibthorpe $^{4}$, Wayne Holland ${ }^{5,6}$, Mark Booth ${ }^{7}$, \\ Paul Kalas $^{8,9}$, Meredith MacGregor $^{10}{ }^{10}$ David Wilner ${ }^{10}$, Bart Vandenbussche $^{11}$, Göran Olofsson ${ }^{12}$, \\ Joris BlommaerT $^{13,14}{ }^{\text {, Alexis BrandeKer }}{ }^{12}$, W. R. F. Dent ${ }^{15}$, Bernard L. De Vries ${ }^{12,16}$, \\ James Di Francesco ${ }^{1,2}$, Malcolm Fridlund ${ }^{17,18}$, James R. Graham ${ }^{8}$, Jane Greaves ${ }^{19}$, Ana M. Heras ${ }^{20}$, \\ Michiel Hogerheijde ${ }^{18}$, R. J. Ivison ${ }^{5,21}$, Eric Pantin ${ }^{22}$, and Göran L. Pilbrati ${ }^{20}$ \\ ${ }^{1}$ National Research Council of Canada Herzberg Astronomy and Astrophysics Programs, 5071 West Saanich Road, Victoria, BC, V9E 2E7, Canada \\ ${ }^{2}$ University of Victoria, 3800 Finnerty Road, Victoria, BC, V8P 5C2, Canada \\ ${ }^{3}$ Institute of Astronomy, University of Cambridge, Madingley Road, Cambridge, CB3 OHA, UK \\ ${ }^{4}$ SRON Netherlands Institute for Space Research, Groningen, The Netherlands \\ ${ }^{5}$ Institute for Astronomy, University of Edinburgh, Royal Observatory, Blackford Hill, Edinburgh, EH9 3HJ, UK \\ ${ }^{6}$ UK Astronomy Technology Centre, Science and Technology Facilities Council, Royal Observatory, Blackford Hill, Edinburgh, EH9 3HJ, UK \\ ${ }^{7}$ Instituto de Astrofsica, Pontificia Universidad Católica de Chile, Vicua Mackenna 4860, 7820436 Macul, Santiago, Chile \\ ${ }^{8}$ Department of Astronomy, University of California, 601 Campbell Hall, Berkeley, CA 94720, USA \\ ${ }^{9}$ SETI Institute, Mountain View, CA 94043, USA \\ ${ }^{10}$ Harvard-Smithsonian Center for Astrophysics, 60 Garden Street, Cambridge, MA 02138, USA \\ ${ }^{11}$ Institute of Astronomy KU Leuven, Celestijnenlaan 200D, B-3001 Leuven, Belgium
${ }^{12}$ Department of Astronomy, AlbaNova University Centre, Stockholm University, SE-106 91 Stockholm, Sweden \\ ${ }^{13}$ Astronomy and Astrophysics Research Group, Department of Physics and Astrophysics, Vrije Universiteit Brussel, Pleinlaan 2, B-1050 Brussels, Belgium \\ ${ }_{15}^{14}$ Flemish Institute for Technological Research (VITO), Boeretang 200, B-2400 Mol, Belgium \\ ${ }^{15}$ Joint ALMA Observatory, Alonso de Cordova 3107, Vitacura 763-0355, Santiago, Chile \\ ${ }^{16}$ Stockholm University Astrobiology Centre, SE-106 91 Stockholm, Sweden \\ ${ }^{17}$ Department of Earth and Space Sciences, Chalmers University of Technology, Onsala Space Observatory, SE-439 92, Onsala, Sweden \\ ${ }^{18}$ Leiden Observatory, Leiden University, Postbus 9513, 2300 RA, Leiden, The Netherlands
School of Physics and Astronomy, University of St. Andrews, North Haugh, St. Andrews, Fife KY16 9SS, UK \\ ${ }^{20}$ ESA, Scientific Support Office, Directorate of Science and Robotic Exploration, European Space Research and Technology Centre (ESTEC/SRE-S), \\ Keplerlaan 1, 2201 AZ Noordwijk, The Netherlands \\ ${ }^{21}$ European Southern Observatory, Karl Schwarzschild Strasse 2, Garching, Germany \\ ${ }^{22}$ Laboratoire AIM, CEA/DSM-CNRS-Universit Paris Diderot, IRFU/SAp, F-91191 Gif sur Yvette, France \\ Received 2015 February 11; accepted 2015 July 31; published 2015 September 28
}

\begin{abstract}
We present far-infrared and submillimeter maps from the Herschel Space Observatory and the James Clerk Maxwell Telescope of the debris disk host star AU Microscopii. Disk emission is detected at 70, 160, 250, 350, 450,500 , and $850 \mu \mathrm{m}$. The disk is resolved at 70, 160, and $450 \mu \mathrm{m}$. In addition to the planetesimal belt, we detect thermal emission from AU Mic's halo for the first time. In contrast to the scattered light images, no asymmetries are evident in the disk. The fractional luminosity of the disk is $3.9 \times 10^{-4}$ and its milimeter-grain dust mass is $0.01 M_{\oplus}( \pm 20 \%)$. We create a simple spatial model that reconciles the disk spectral energy distribution as a blackbody of $53 \pm 2 \mathrm{~K}$ (a composite of 39 and $50 \mathrm{~K}$ components) and the presence of small (non-blackbody) grains which populate the extended halo. The best-fit model is consistent with the "birth ring" model explored in earlier works, i.e., an edge-on dust belt extending from 8.8 to $40 \mathrm{AU}$, but with an additional halo component with an $r^{-1.5}$ surface density profile extending to the limits of sensitivity (140 AU). We confirm that AU Mic does not exert enough radiation force to blow out grains. For stellar mass-loss rates of 10-100 times solar, compact (zero porosity) grains can only be removed if they are very small; consistently with previous work, if the porosity is 0.9 , then grains approaching $0.1 \mu \mathrm{m}$ can be removed via corpuscular forces (i.e., the stellar wind).
\end{abstract}

Key words: circumstellar matter - stars: individual (AU Mic)

\section{INTRODUCTION}

Debris disks are one of the most prevalent signposts that a stellar system succeeded in building up planetary scale bodies during the protoplanetary disk phase. Debris disks are collisionally sustained distributions of planetesimals, smaller rocky bodies, and dust around main-sequence (and more evolved) stars. Because dust grains can be removed from the system through various physical processes, their presence is direct evidence of an unseen population of larger planetesimals, and potentially planets, in orbit around the star. At current sensitivity levels, debris disks are found around $\gtrsim 20 \%$ of nearby solar and A-type stars (Eiroa et al. 2013; Matthews et al. 2014b; Thureau et al. 2014; B. C. Matthews et al. 2015, in preparation), with enhanced detection rates around younger stars, e.g., the
Pleiades: Gorlova et al. (2006) find 25\% for B- and A-type stars while Sierchio et al. (2010) find 32\% for solar types; the 10-20 Myr old Sco-Cen association: Chen et al. (2011, 2012) find rates from $25 \%$ to $33 \%$, varying with spectral type; and ensembles of A star populations: Su et al. (2006) find a rate of $32 \%$ at 24 and $70 \mu \mathrm{m}$. There is, however, a relative paucity of disks detected around M stars (Matthews et al. 2014b). Recent surveys for debris disks have revealed very low detection rates for $\mathrm{M}$ star hosted debris disks compared to earlier type stars (Low et al. 2005; Gautier et al. 2007). For example, data from the DEBRIS survey with the Herschel Space Observatory ${ }^{23}$ reveal

\footnotetext{
$\overline{23}$ Herschel is an ESA space observatory with science instruments provided by European-led Principal Investigator consortia and with important participation from NASA.
} 
just two disks in a sample of 89 observed M stars within $8.6 \mathrm{pc}$ (B. C. Matthews et al. 2015, in preparation). The mass sensitivity to $\mathrm{M}$ star disks in existing surveys, however, has not yet matched those of earlier spectral types (see Figure 2 of Matthews et al. 2014b).

Several factors affect the detectability of disks around $\mathrm{M}$ stars. For example, grain removal by stellar winds is more efficient around $M$ stars (e.g., Plavchan et al. 2005), suggesting that small grains (i.e., $<1 \mu \mathrm{m})$ may be removed from these systems at a rate higher than expected purely from radiation forces (Augereau \& Beust 2006; Matthews et al. 2007). This effect may explain why the highest detection rate of $13_{-8}^{+6} \%$ is found for a combination of submillimeter studies since these are sensitive to larger grains (Lestrade et al. 2006). Forbrich et al. (2008) find that for the 30-40 Myr old cluster NGC 2547, the detection rates of $\mathrm{M}$ star disks at $24 \mu \mathrm{m}$ exceeds that of $\mathrm{G}$ and $\mathrm{K}$ stars of the same age, suggesting that at least around very young stars, $\mathrm{M}$ star disks may be just as detectable as disks around earlier type stars, consistent with the bright disk detected around the M3IVe (Pecaut \& Mamajek 2013) star AU Mic, the M3IVe (Pecaut \& Mamajek 2013) star TWA 7's disk (Matthews et al. 2007), and the recently identified disk (Kennedy et al. 2014) around the M4 star Fomalhaut C (Mamajek et al. 2013). Nevertheless, a disk of high fractional luminosity has also been detected and resolved around the significantly older, multiple planet host GJ 581 (Lestrade et al. 2012). Therefore, AU Mic, as the first and youngest nearby $M$ star to have a detected disk, retains particular importance as a representative of its class.

AU Mic (GJ 803, HD 197481) was the only M star detected to have a far-IR excess above its stellar photosphere (indicative of circumstellar dust) with IRAS (Faint Source Catalog, Moshir et al. 1990). A submillimeter excess was detected with photometric-mode observations with the SCUBA camera, and the emission was not resolved in a relatively shallow mapping observation (Liu et al. 2004). An excess was also seen with the $\mathrm{CSO}$ at $350 \mu \mathrm{m}$ (Chen et al. 2005). Ground-based imaging by Kalas et al. (2004) and subsequent high-resolution imaging and polarimetry from the Hubble Space Telescope by Krist et al. (2005) and Graham et al. (2007) revealed it to be the second debris disk spatially resolved at optical wavelengths, hosting an edge-on disk that extends to over $100 \mathrm{AU}$ in radius. The Graham et al. (2007) HST polarization study revealed evidence of a change in the polarization properties at a radius of $\sim 35 \mathrm{AU}$ and a dearth of micron-sized grains interior to $40 \mathrm{AU}$. Wilner et al. (2012) imaged the disk with the SubMillimeter Array (SMA) and most recently, imaging with the Atacama Large Millimeter/submillimeter Array (ALMA) yielded a wellresolved disk image modeled as a narrow "birth ring" or "parent belt" of planetesimals at $40 \mathrm{AU}$ (MacGregor et al. 2013). The ALMA data do suggest a reasonably wide dust belt extending inward to $\sim 9$ AU though the dust surface density is strongly peaked near $40 \mathrm{AU}$ and the inner edge is poorly constrained. In recent STIS observations, Schneider et al. (2014) report an "out-of-plane bump" on one side of the disk at $\sim 13 \mathrm{AU}$, interpreting this as a dust density enhancement, in contrast to the ALMA data that revealed no significant asymmetries. Schneider et al. (2014) also observe a brightness asymmetry between the two sides of the scattered light disk interior to 15-20 AU, a region that had not been cleanly imaged in earlier scattered light detections. Finally, the ALMA data also revealed an unresolved excess at the position of the star which MacGregor et al. note could be attributable to unresolved emission from an asteroid-like warm belt near the star. Cranmer et al. (2013), however, suggest that coronal thermal heating could alternatively account for the observed excess at the position of the star.

AU Mic attains additional importance as a member of the Beta Pictoris Moving Group since, as such, it has a relatively well-established young age of $23 \pm 3 \mathrm{Myr}$ (Binks \& Jeffries 2014; Malo et al. 2014; Mamajek \& Bell 2014), revised upward from $12_{-4}^{+8} \mathrm{Myr}$ (Zuckerman et al. 2001). Due to its youth and proximity (9.91 \pm 0.10 pc, van Leeuwen 2007), AU Mic is a favored target for study. As described in detail by Wilner et al. (2012), its disk also shares many qualitative characteristics with the $\beta$ Pictoris disk, including its edge-on geometry and extended scattered light emission (Kalas et al. 2004). The age of AU Mic is the currently favored epoch for the formation of terrestrial planets (Raymond et al. 2012; Chambers 2014). While M stars do host planetary systems, including the multiple planets around GJ 876 (Rivera et al. 2005), GJ 581 (Udry et al. 2007), and GJ 676A (AngladaEscudé \& Tuomi 2012). No planets have been detected around AU Mic, though Schneider et al. (2014) do suggest a potential planetary origin for the observed asymmetry in scattered light.

AU Mic is similar to other bright debris disks in that it possesses a bright "halo," seen in scattered light images. In debris disk systems, where the smallest grains are blown out of the system as soon as they are created, and larger grains are unaffected, there necessarily exists an intermediate size range where newly created particles are placed on eccentric orbits. The specific sizes of these particles depends on the stellar luminosity and mass-loss rate, but in general they are smaller than $10 \mu \mathrm{m}$. These particles have pericenters within the parent belt (or birth ring), but apocenters extending to the maximum allowed by the local environment. Thus, they form a smallgrain "halo" that surrounds the parent belt. In the case of AU Mic, the radiation force is relatively weak and the halo is created by the radial force exerted on the dust by the stellar wind, which is thought to be 10-100 times greater than the Solar wind (Augereau \& Beust 2006; Strubbe \& Chiang 2006). A key prediction is therefore that halos should be relatively faint at millimeter wavelengths where the small grains emit inefficiently, and thus only the parent belt is detected. This hypothesis has been confirmed by millimeter-wave observations of AU Mic (Wilner et al. 2012; MacGregor et al. 2013), as well as for other systems (e.g., Vega, $\beta$ Pic, and HR 8799; Holland et al. 1998; Dent et al. 2014; Williams \& Andrews 2006, respectively), thus explaining how debris disks can have different apparent sizes at different wavelengths. For both Vega and HR 8799, the halo has only been detected in mid/far-IR emission ( $\mathrm{Su}$ et al. 2009; Sibthorpe et al. 2010; Matthews et al. 2014a).

We present the first resolved far-infrared images of AU Mic's debris disk from the Herschel Space Observatory's PACS (Photodetector Array Camera and Spectrometer, Poglitsch et al. 2010) instrument at 70 and $160 \mu \mathrm{m}$ and lower resolution submillimeter images from the SPIRE (Spectral and Photometric Imaging REceiver, Griffin et al. 2010) camera at 250, 350, and $500 \mu \mathrm{m}$. These data were taken during Guaranteed Time, as part of the Disk Evolution Key Program (PI: Olofsson). In addition, we present 850 and $450 \mu \mathrm{m}$ data from the SCUBA-2 Observations of Nearby Stars (SONS) Survey and a PI program, taken with the SCUBA-2 camera (Holland et al. 2013). We 
Table 1

Observations Log

\begin{tabular}{|c|c|c|c|c|}
\hline Obs. ID & Observing Date & Mode & Duration (s) & PWV (mm) \\
\hline \multicolumn{5}{|c|}{ Herschel Space Observatory } \\
\hline 1342196038 & 2010 May 9 & PacsPhoto 70/160 (scan 135) & 5478 & $\cdots$ \\
\hline 1342196103 & 2010 May 9 & PacsPhoto 70/160 (scan 45) & 5478 & $\cdots$ \\
\hline 1342193786 & 2010 Apr 5 & SpirePhoto & 2906 & $\cdots$ \\
\hline \multicolumn{5}{|c|}{ James Clerk Maxwell Telescope } \\
\hline \multirow[t]{6}{*}{ SCUBA-2 } & 2012 Apr 22 & daisy scan $850 / 450$ & 3910 & 1.06 \\
\hline & 2012 Apr 23 & daisy scan $850 / 450$ & 3947 & $0.83-0.92$ \\
\hline & 2012 May 17 & daisy scan $850 / 450$ & 1960 & 0.92 \\
\hline & 2012 Jun 7 & daisy scan $850 / 450$ & 1895 & 1.4 \\
\hline & 2012 Aug 16 & daisy scan $850 / 450$ & 3787 & 0.86 \\
\hline & 2012 Aug 19 & daisy scan $850 / 450$ & 3790 & 0.83 \\
\hline
\end{tabular}

describe our observations and data reduction in Section 2 and present the data in Section 3. In Section 4, we discuss the new constraints these data place on models of the disk emission, namely the temperature of the planetesimal belt and halo and the extent of the halo in thermal emission. We summarize our conclusions in Section 5.

\section{OBSERVATIONS}

A summary of details of the observations from Herschel (Pilbratt et al. 2010) and the James Clerk Maxwell Telescope (JCMT) is shown in Table 1 . The relative sensitivities of Spitzer/MIPS24, Herschel/PACS, and SCUBA-2 observations to the AU Mic disk in terms of fractional luminosity $\left(L_{\text {disk }} / L_{\star}\right)$ against blackbody radial dust location are shown in Figure 1.

\subsection{Herschel Observations}

Large area photometric mapping observations were performed using the PACS and SPIRE cameras at 70, 160, 250, 350 , and $500 \mu \mathrm{m}$. The large scan-map mode was used and nearly perpendicular cross-linked scans were performed with both instruments. The total observing time was $3.8 \mathrm{hr}$. The images at each observed wavelength are shown in Figure 2.

The PACS observations used scan legs of 7!.4 with a scanleg spacing of $38^{\prime \prime}$. Medium scan rate maps containing 11 scan legs were repeated 11 times in each scanning direction to achieve the required map depth. Data were obtained at both 70 and $160 \mu \mathrm{m}$ simultaneously.

The nominal cross-linked large map observing parameters were used for the SPIRE observations, with a smaller map of 4 arcmin by 4 arcmin being executed. In total, 10 repeat maps were used to reach the required depth.

The data were processed using version 13.0 of the Herschel interactive pipeline environment (HIPE, Ott 2010). The standard pipeline processing steps were used for both the PACS and SPIRE data. Versions 69 and 13.1 of the PACS and SPIRE calibration products were applied respectively. The final maps have pixel scales of $1,2,6,10$, and $14^{\prime \prime}$ at $70,160,250$, 350 , and $500 \mu \mathrm{m}$ respectively.

As part of the data processing, the PACS timelines were first masked at the source location, as well as in other areas of bright emission, and then high-pass filtered to reduce the impact of 1/ $f$ noise. The filtered data were then converted to maps using the "photProject" task. Maps were likewise made from the SPIRE

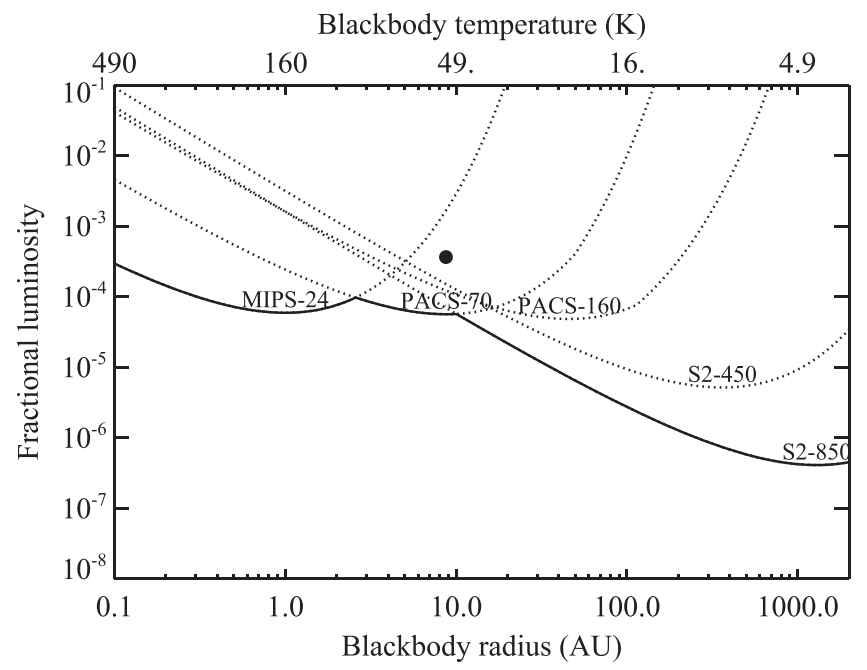

Figure 1. Relative sensitivities of Spitzer MIPS $(24 \mu \mathrm{m})$, Herschel PACS, and SCUBA-2 to dust emission at the level of AU Mic's disk, shown as a black dot. Any disk above each instrument's sensitivity curve will be detectable to that instrument. The best-fit temperature and fractional luminosity of AU Mic render undetectable to MIPS at $24 \mu \mathrm{m}$ (as reported by Chen et al. 2005), but detectable to PACS at 70 and $160 \mu \mathrm{m}$ as well as SCUBA-2 at 450 and $850 \mu \mathrm{m}$.

data using the "naiveMapper" task. No filtering was performed on the SPIRE data.

Observations of $\alpha$ Tau and Neptune, adjusted to the correct spacecraft position angle, were used as the model PACS and SPIRE point-spread functions (PSFs) respectively, and used for model image convolution.

\subsection{JCMT Observations}

Observational data presented in this paper were also taken using the SCUBA-2 camera (Holland et al. 2013) on the JCMT. The data were obtained both as part of the SONS JCMT Legacy survey (Phillips et al. 2010) and a PI program. The observations were taken with the constant speed DAISY pattern (Holland et al. 2013), which maximizes exposure time and provides uniform coverage in the central $3^{\prime}$ diameter region of a field. The total integration time was just over $5 \mathrm{hr}$, split into 10 separate $\sim 30$ minute observations. Observing conditions were generally excellent with precipitable water vapour levels less than $1 \mathrm{~mm}$, corresponding to zenith sky opacities of around 1.0 and 0.2 at 450 and $850 \mu \mathrm{m}$ respectively (equivalent to 

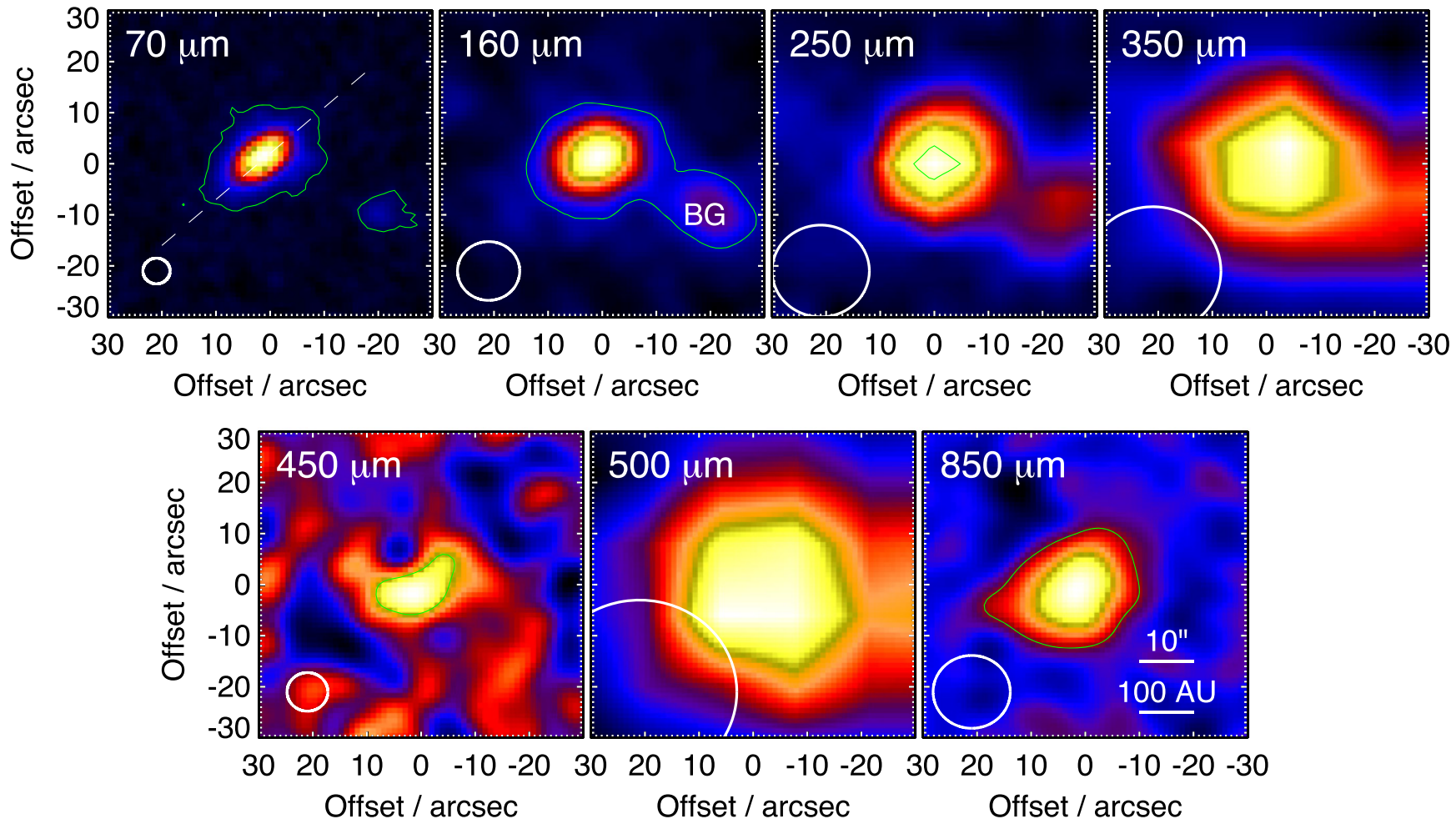

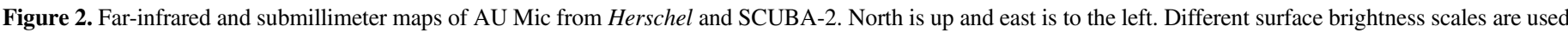

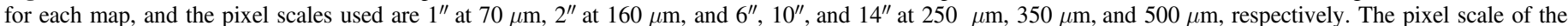

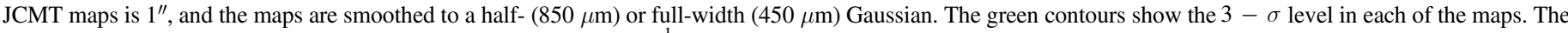

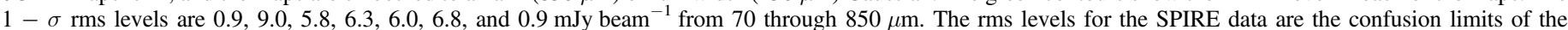

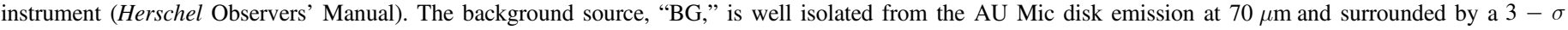
contour at 70 and $160 \mu \mathrm{m}$ (labeled).

JCMT weather "grade 1"; $\tau_{225 \mathrm{GHz}}$ of $\left.<0.05\right)$. The data were calibrated in flux density against the primary calibrator Uranus and also secondary calibrators CRL 618 and CRL 2688 from the JCMT calibrator list (Dempsey et al. 2013), with estimated calibration uncertainties amounting to $10 \%$ at $450 \mu \mathrm{m}$ and $5 \%$ at $850 \mu \mathrm{m}$.

The SCUBA-2 data were reduced using the Dynamic Iterative Map-Maker within the STARLINK SMURF package (Chapin et al. 2013) called from the ORAC-DR automated pipeline (Cavanagh et al. 2008). The map maker used a configuration file optimized for known position, compact sources. It adopts the technique of "zero-masking" in which the map is constrained to a mean value of zero (in this case outside a radius of $60^{\prime \prime}$ from the center of the field), for all but the final interation of the map maker (Chapin et al. 2013). The technique not only helps convergence in the iterative part of the map-making process but suppresses the large-scale ripples that can produce ringing artefacts. The data are also high-pass filtered at $1 \mathrm{~Hz}$, corresponding to a spatial cut-off of $\sim 150^{\prime \prime}$ for a typical DAISY scanning speed of $155^{\prime \prime} \mathrm{s}^{-1}$. The filtering removes residual low-frequency (large spatial scale) noise and, along with the "zero-masking" technique, produces flat, uniform final images largely devoid of gradients and artefacts (Chapin et al. 2013).

To account for the attenuation of the signal as a result of the time series filtering, the pipeline re-makes each map with a fake 10 Jy Gaussian added to the raw data, but offset from the nominal map center by $30^{\prime \prime}$ to avoid contamination with any detected source. The amplitude of the Gaussian in the output map gives the signal attenuation, and this correction is applied along with the flux conversion factor derived from the calibrator observations. The final images were made by coadding the 10 maps using inverse-variance weighting, regridded with 1 arcsec pixels at both wavelengths. The final images at both wavelengths have been smoothed with a 7 " FWHM Gaussian to improve the signal-to-noise ratio. The FWHMs of the primary beam are $7 " .9$ and $13^{\prime \prime} .0$ at $450 \mu \mathrm{m}$ and $850 \mu \mathrm{m}$, respectively.

\section{RESULTS}

Figure 2 shows the observed thermal emission on the sky toward AU Mic at seven wavelengths. The maps have been cropped to show only a $1^{\prime} \times 1^{\prime}$ field centered on the star. AU Mic is detected at all wavelengths. The disk is resolved at 70 and $160 \mu \mathrm{m}$ with Herschel. Two-dimensional Gaussian fits to the JCMT data yield sizes of $16^{\prime \prime} 0 \times 8$." $^{\prime \prime}$ at $450 \mu \mathrm{m}$ (at $\mathrm{PA} \approx 135^{\circ}$, aligned with the scattered light images) and 16 . $9 \times 14$." 4 at $850 \mu \mathrm{m}$, which suggest that the source is resolved along the major axis at $450 \mu \mathrm{m}$ (as also indicated by the difference in the peak and integrated flux densities in Table 2) but only marginally at $850 \mu \mathrm{m}$ (see composite Figure 3).

The PACS flux densities were measured using aperture photometry, with radii of $17^{\prime \prime}$ at $70 \mu \mathrm{m}$ and $34^{\prime \prime}$ at $160 \mu \mathrm{m}$. We used aperture corrections of 0.81 and 0.85 at 70 and $160 \mu \mathrm{m}$, which were derived from a large set of calibration observations processed in the same way as the data. At $160 \mu \mathrm{m}$, this aperture includes the background source, whose point source flux was estimated in the modeling described below and has been 
Table 2

Measured Fluxes

\begin{tabular}{|c|c|c|c|c|c|}
\hline $\begin{array}{l}\text { Wavelength } \\
(\mu \mathrm{m})\end{array}$ & $\begin{array}{c}\text { Peak Flux } \\
\left(\mathrm{mJy} \mathrm{beam}^{-1}\right)\end{array}$ & $\begin{array}{l}\text { Integrated Flux } \\
\text { (mJy) }\end{array}$ & $\begin{array}{l}F_{\mathrm{BG}} \\
(\mathrm{mJy})\end{array}$ & $\begin{array}{l}F_{\text {phot }} \\
(\mathrm{mJy})\end{array}$ & $\begin{array}{l}\text { Disk Flux } \\
(\mathrm{mJy})\end{array}$ \\
\hline 70 & $91.6 \pm 2.7$ & $231 \pm 7$ & $11.6 \pm 0.35$ & 19.6 & $219 \pm 7$ \\
\hline 160 & $176 \pm 8.8$ & $228 \pm 15$ & $47.1 \pm 2.3$ & 3.6 & $226 \pm 15$ \\
\hline 450 & $32.3 \pm 5.5$ & $49.2 \pm 8.5$ & non-detection & 0.44 & $35.4 \pm 8.5$ \\
\hline 850 & $12.9 \pm 0.9$ & $14.0 \pm 1.5$ & non-detection & 0.10 & $12.5 \pm 1.5$ \\
\hline
\end{tabular}

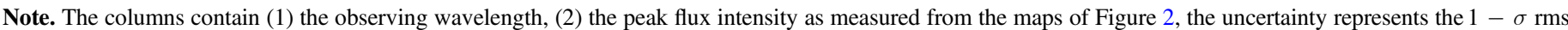

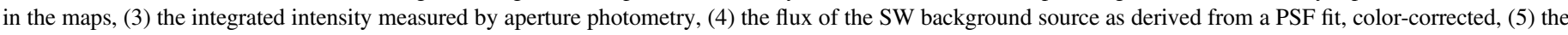

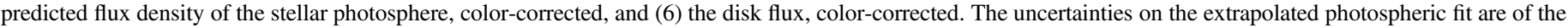
order of a few percent and therefore negligible compared with the measured uncertainties.

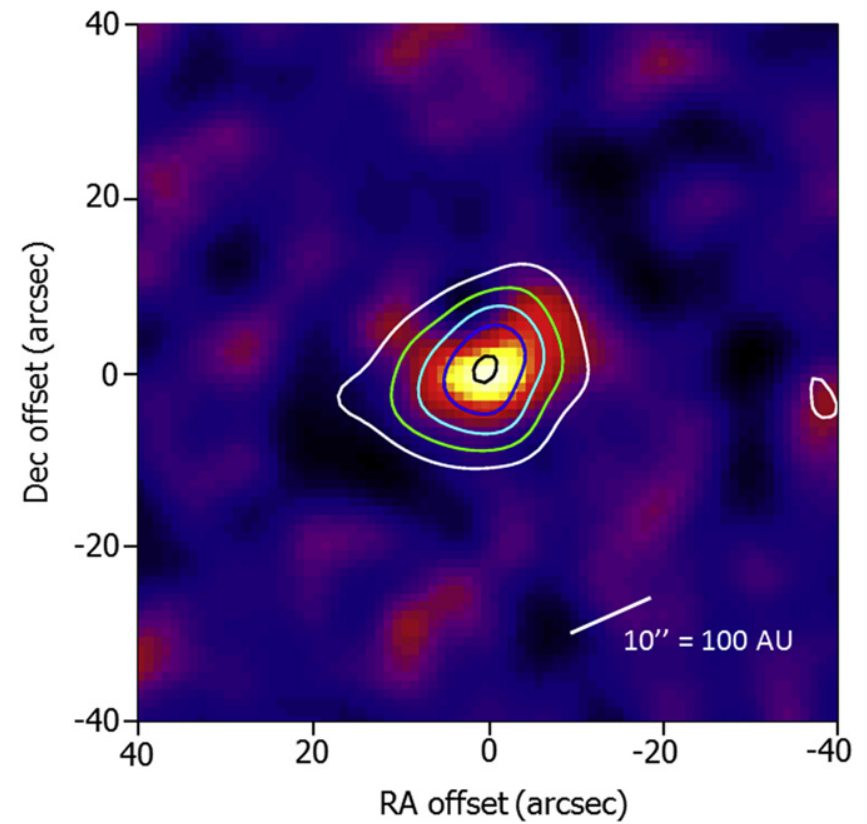

Figure 3. SCUBA-2 imaging of the AU Mic disk. North is up and east is to the left. Composite image showing the extension of the disk along the known disk orientation (shown by the orientation of the scale bar) at $450 \mu \mathrm{m}$ in grayscale with an overlay of the $850 \mu \mathrm{m}$ contours. The contour levels are rms levels of $3 \sigma$ (white), $6 \sigma$ (green), $9 \sigma$ (light blue), $12 \sigma$ (blue), and $15 \sigma$ (black).

subtracted to estimate the AU Mic flux density alone. The rms noise levels were estimated by integrating the flux within beam-sized circular apertures $(\sim 10$ at $70 \mu \mathrm{m})$, spaced by the FWHM beam, over the central $2^{\prime}$ diameter area. However, the PACS flux densities have an uncertainty that is largely set by the instrumental calibration, with some additional uncertainty at $160 \mu \mathrm{m}$ to allow for subtraction of the background source. At 70 and $160 \mu \mathrm{m}$, the absolute flux calibration accuracies are 3\% and 5\% respectively (Herschel Observers' Manual; Balog et al. 2014). Due to the depth of the image, the measurement errors are negligible compared to the calibration errors, so the combined residual sum of squares uncertainties are the same as the calibration errors. We attempted to measure SPIRE flux densities using PSF fits. Our flux extraction at SPIRE wavelengths, however, is severely impacted by our inability to separate the flux density of the AU Mic disk from nearby background objects, so we do not quote any fluxes. The JCMT flux density measurements were made using $20^{\prime \prime}$ radius apertures, with the same method for uncertainty estimation.

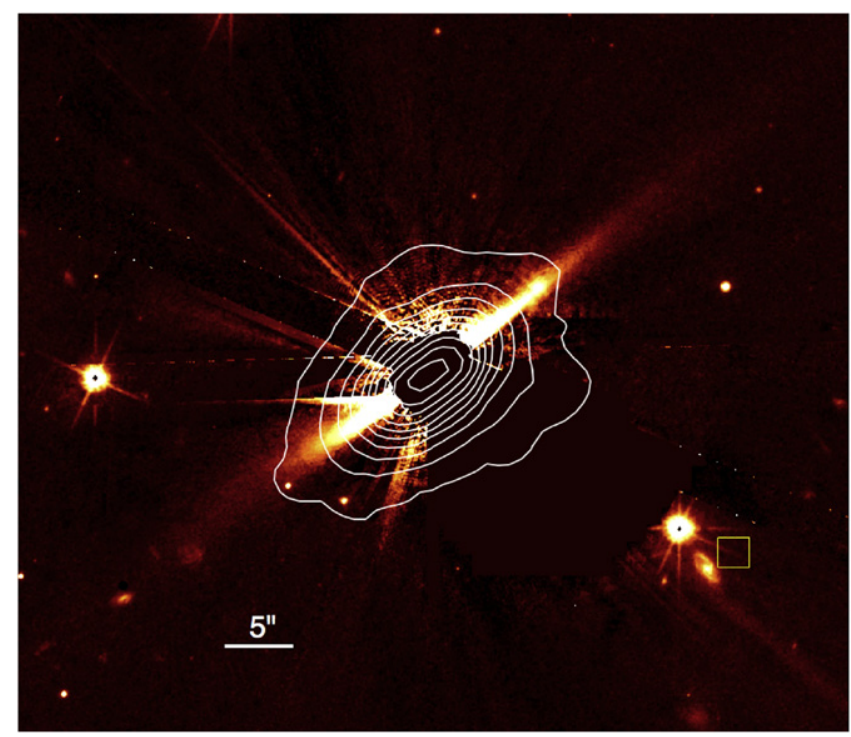

Figure 4. Optical image of AU Mic from $H S T$ showing the orientation and extent of the scattered light disk. North is up and east is to the left. The $70 \mu \mathrm{m}$ emission from Herschel is overlaid. The disk is clearly resolved at $70 \mu \mathrm{m}$, and the disk orientations are consistent, to the limits of the Herschel resolution. The yellow box shows the position of the $70 \mu \mathrm{m}$ source detected with Herschel at the epoch of the HST observations. The position is close to, but not coincident with, the optically visible galaxy approximtely $2^{\prime \prime}$ to the southeast.

At these wavelengths, however, the calibration uncertainty $(\sim 10 \%)$ is relatively unimportant.

Table 2 reports the peak flux densities and integrated flux densities of AU Mic at each observing wavelength. Furthermore, we list the PSF-fit flux density of the "BG" background source identified in Figure 2. These measurements are found to be in good agreement with those derived from the image model of AU Mic described below.

Rebull et al. (2008) report flux densities of $143 \pm 2 \mathrm{mJy}$ at $24 \mu \mathrm{m}, 205 \pm 8 \mathrm{mJy}$ at $70 \mu \mathrm{m}$, and $168 \pm 20$ at $160 \mu \mathrm{m}$. Plavchan et al. (2009) report updated flux densities of $155.2 \pm$ 3.2 and $223 \pm 26 \mathrm{mJy}$ at 24 and $70 \mu \mathrm{m}$. The photospheric flux at $24 \mu \mathrm{m}$ is $150 \pm 2 \mathrm{mJy}$ so there is no significant excess at this wavelength. Our measured flux density at $70 \mu \mathrm{m}$ from Herschel is consistent with the measured Spitzer flux density, while we measure a higher flux density (but consistent within $2 \sigma$ ) than Spitzer at $160 \mu \mathrm{m}$.

The only significant source of emission within $1^{\prime}$ of AU Mic is located at P.A. $=244.0$ and separation $\sim 25^{\prime \prime}$. To determine if this is a background galaxy, we checked the HST F814W observations made with ACS/WFC in 2004 (GO-10228; PI 


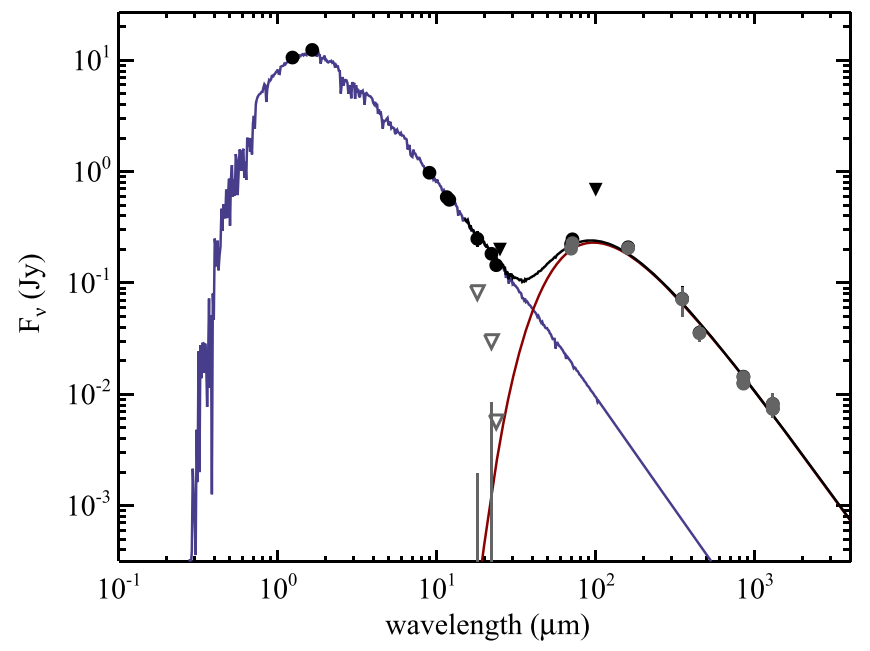

Figure 5. SED of AU Mic and its disk. The fit to the stellar photosphere is given in blue; the disk fit is shown in red, and the composite stellar + disk spectrum is shown in black. Black symbols are observed flux densities and gray symbols show star-subtracted values. Inverted triangles show upper limits (the $100 \mu \mathrm{m}$ value is from IRAS). In the absence of resolved imaging, a single component disk would be a reasonable interpretation of this SED, for which the excess emission is well characterized by a pure blackbody.

Kalas). Figure 4 shows the $70 \mu \mathrm{m}$ Herschel contours overlaid on the HST image. No galaxy is detected in these optical data that corresponds to the $70 \mu \mathrm{m}$ source to the southwest of $\mathrm{AU}$ Mic (position at epoch of optical data is given by the yellow box), although the galaxy in the optical image is quite close ( $\sim 2^{\prime \prime}$, the pointing accuracy of Herschel). The absence of a coincident galaxy is not unexpected given the relative sensitivity to infrared faint galaxies of Herschel compared to optical observations.

The flux density distribution (which we loosely call the spectral energy distribution, or SED) of AU Mic and its disk is shown in Figure 5. To derive the stellar spectrum, we compiled $U B V$, Hipparcos/Tycho-2, 2MASS, Spitzer, AKARI, and $W I S E$ photometry up to $12 \mu \mathrm{m}$. We excluded the $U-B$ color to avoid potential issues with variability due to flaring at the shortest wavelengths. The best fit to the BT-Settl stellar atmosphere models (Allard et al. 2012) yields $T_{\text {eff }}=3600 \pm 20 \mathrm{~K}$, a radius of $0.83 R_{\odot}$, and a luminosity of $0.1 L_{\odot}$. Low-res IRS Spitzer data range from 5 to $14 \mu \mathrm{m}$ and are consistent with the stellar photosphere, but not shown in Figure 5 . $^{24}$

We then subtracted the photospheric model from the flux densities at longer wavelengths, and fitted a pure blackbody to this star-subtracted photometry. The resulting model is shown in Figure 5, where the disk has a temperature of $53 \pm 2 \mathrm{~K}$ and a fractional luminosity of $3.5 \times 10^{-4}$. That the disk spectrum is well fit by a pure blackbody is surprising for two reasons: first, most debris disks have an emission spectrum steeper than Rayleigh-Jeans at long wavelengths (e.g., Wyatt 2008; Gáspár et al. 2012), and second, the disk is well known to be extended from scattered light imaging (Kalas et al. 2004; Liu 2004; Krist et al. 2005; Metchev et al. 2005; Fitzgerald et al. 2007; Graham et al. 2007; Schneider et al. 2014), and thus must comprise dust at a wide range of temperatures. The goal of the spatial modeling in the next section is to use a simple model to reconcile the disk extent and blackbody spectrum.

\footnotetext{
${ }^{24}$ A high-resolution IRS Spitzer scan was examined but was not useable.
}

\subsection{Modeling}

We use a simple model to interpret the Herschel, SCUBA-2, and ALMA observations. Preliminary tests find that the Herschel $70 \mu \mathrm{m}$ image is significantly more extended than the parent belt imaged with ALMA at $1.3 \mathrm{~mm}$, so the basic requirement of the model is that it reconciles the different extent of these images and produces a blackbody-like spectrum. As is already known, the solution is that the greater radial extent at $70 \mu \mathrm{m}$ originates in the halo of small grains also seen in scattered light, and that these are not seen with ALMA because these grains emit inefficiently at wavelengths significantly larger than their physical size. Thus, our model comprises two components; the first is the parent bodies for which we use the ALMA modeling results of MacGregor et al. (2013), and the second is a halo whose basic properties are to be informed by previous theory work and determined from the modeling.

With only six photometric points in the spectrum, and a only a few beams of resolution in the new Herschel and JCMT images, our modeling approach is physically motivated but simple. It has been used previously to model many Herschelresolved debris disks (e.g., Kennedy et al. 2012a, 2012b; Matthews et al. 2014a). For each disk component, a single azimuthally symmetric 3D dust distribution is used, which is simply a small-scale height disk with a power-law surface density dependence between the inner and outer radii. At each radial location, the disk emission is assumed to arise from a modified blackbody, with a power-law radial temperature dependence. This approach is therefore largely empirical, and the derived blackbody parameters can be subsequently compared with more detailed dust models to draw conclusions about the dust properties, in particular, their typical size. The limited resolution also precludes derivation of comparative radial profiles, as was done for HR 8799's halo (Matthews et al. 2014a).

Practically, we generate high-resolution images at each observed wavelength, as viewed from a specific direction to produce the desired disk geometry. We can include an arbitrary number of disk components, as well as point sources to model unresolved sources such as warm inner disk regions or background sources. Each image is then convolved with the appropriate PSF, which may be an observation of a bright calibration source (i.e., PACS, SPIRE), or simply a Gaussian with specified width(s) and position angle (i.e., JCMT, ALMA), and then resampled to the resolution of the observation. We model the ALMA image and not the visibilities, which is acceptable given the good $u v$ coverage and dynamic range of the ALMA data. The models are fit to the data using a combination of by-eye variation of parameters and least-squares minimization. Since we do not search all parameter space, our best-fit models are not necessarily unique, but must be considered a reasonable interpretation.

Our model is guided by previous modeling work. The parent belt is modeled using the structure derived by MacGregor et al. (2013) based on ALMA data, here using a surface density distribution between 8.8 and $40 \mathrm{AU}$ with a radial power law dependence of $\Sigma \propto r^{2.8}$. The inner edge is not tightly constrained. MacGregor et al. find that the inner edge of the parent belt could be as far out as $21 \mathrm{AU}$, suggesting a much narrower disk width, though we note that Schneider et al. (2014) find evidence of a stello-symmetric warp in the disk with an outer edge of $15 \mathrm{AU}$, which supports the idea that some 
component of the disk has an inner edge closer than 21 AU. Note that we are calling this component the parent belt for convenience, and that the extent inward of 40 AU may be due to stellar wind drag from a true source region that is much narrower, but likely still concentrated at $\sim 40$ AU. The emission properties of grains in this belt are assumed to be blackbodylike, and follow $T_{\mathrm{PB}}=T_{0, \mathrm{~PB}} / \sqrt{r}$, meaning that the surface brightness profile matches the power-law found by MacGregor et al. (2013). Because radiation and wind forces are relatively weak for AU Mic, small dust can reside in the planetesimal belt and $T_{0, \mathrm{~PB}}$ can be higher than expected for blackbodies, so we leave it as a free parameter. We also add the unresolved point source component at the stellar position seen in the ALMA data (MacGregor et al. 2013), though this source is too faint to be detected at any other wavelength (see Section 3.2).

To reproduce the Herschel $70 \mu \mathrm{m}$ image, we include the halo component, with a surface density profile fixed to $\Sigma \propto r^{-1.5}$ (e.g., Strubbe \& Chiang 2006), which extends from $40 \mathrm{AU}$ to $140 \mathrm{AU}$. (The outer edge is poorly constrained by the data and fixed at 140 AU.) The surface density of this component is forced to join smoothly to the planetesimal belt, and the grain properties are allowed to vary via their temperatures and emission spectra. Their spectra are modified blackbodies (i.e., a Planck function multiplied by $\left(\lambda_{0} / \lambda\right)^{\beta_{\lambda}}$ for $\left.\lambda>\lambda_{0}\right)$, but we reduce the number of parameters by enforcing a temperature law suited for small grains, $T_{\text {halo }}=T_{0, \text { halo }} r^{-1 / 3}$. We vary $\lambda_{0}, \beta_{\lambda}$, and $T_{0 \text {,halo }}$ as free parameters. As noted above, we expect $\lambda_{0} \lesssim 100 \mu \mathrm{m}$ and $\beta_{\lambda}>0$, so that the halo is detected at $70 \mu \mathrm{m}$, but not at $1.3 \mathrm{~mm}$ with ALMA. These values are consistent with measured values of $\lambda_{0}$ and $\beta$ for other disks (i.e., Booth et al. 2013; Matthews et al. 2014a).

Finally, we include the point source visible to the west of AU Mic in Figure 2. This source is included in part to ensure it does not bias the model at $160 \mu \mathrm{m}$, but is also used to derive a flux density to subtract from the $160 \mu$ m aperture flux derived above. We also include the central point source seen in the $1.3 \mathrm{~mm}$ ALMA image. With this simple model, we have four main parameters; one for the parent belt temperature and three for the halo component temperature and spectrum. There are in addition eight R.A./decl. offsets for the Herschel, JCMT, and ALMA images, but these are relatively well constrained. The aim of the model fitting is therefore essentially to find the relative weight of the two components at each wavelength, thereby empirically deriving a coarse spectrum for each in a way that is independent of assumptions about grain properties. We found that the relatively poor resolution (excepting the ALMA data) means that the parameters are poorly constrained due to degeneracies, in particular for the halo component. However, all solutions we found are sufficiently similar that the ultimate interpretation is the same.

Figure 3 shows the original images, the parent belt and halo model components, and the model-subtracted residuals (for just the planetesimal belt and the full model) at wavelengths of 70 , 160,450 , and $1300 \mu \mathrm{m}$. First, to make it clear that the halo is detected, the fourth column shows the data with only the parent belt subtracted. In this column, the brightness of the parent belt is 1.8 and 1.4 times brighter than in the final model at 70 and $160 \mu \mathrm{m}$. The $70 \mu \mathrm{m}$ residuals clearly show that the disk is more extended than the parent belt, and that a component with greater extent (i.e., the halo) is required. The fifth (rightmost) column shows the residuals with the addition of this component, and that our parent belt + halo model reproduces the data well. Thus, the halo contributes significant emission at $70 \mu \mathrm{m}$, but little at longer wavelengths.

At all wavelengths, few residuals above the $3 \sigma$ level are seen. The negative residuals at $160 \mu \mathrm{m}$ appear to lie along the scan directions (at roughly $\pm 45^{\circ}$ relative to $N$ ) suggesting that they are artefacts of the imaging. The positive residual to the SE lies beyond the disk extent and is not seen on the opposite side of the star, so should not be considered as a deficiency of the model. The ALMA residual map has a bright point source offset from the stellar position. This is a background source identified by MacGregor et al. (2013) as well. It is located 0!' 3 west and 1". 8 south of the star and is detected with a flux density of $0.15 \mathrm{mJy}$ (a $5 \sigma$ detection).

The main derived model parameters are $T_{0, \mathrm{~PB}}=245 \mathrm{~K}$, $T_{0 \text {,halo }}=161 \mathrm{~K}, \lambda_{0}=12 \mu \mathrm{m}$, and $\beta_{\lambda}=1$. Because the structure of the parent belt is fixed, $T_{0, \mathrm{~PB}}$ is moderately well constrained by the contribution required at $70 \mu \mathrm{m}$. The halo properties, which are our primary interest, are less well constrained because is it only strongly detected at $70 \mu \mathrm{m}$. However, the conclusion that $\lambda_{0}$ is shorter than $\sim 70 \mu \mathrm{m}$, and that $\beta_{\lambda} \gtrsim 1$ is robust because the halo is required by the images to have little contribution at $160 \mu \mathrm{m}$ and beyond. Given the conclusions of previous studies that the halo is populated by small dust on eccentric orbits these parameters are consistent with our expectations. The specific value of $\lambda_{0}=12 \mu \mathrm{m}$ suggests that $\sim 1 \mu \mathrm{m}$ sized grains dominate the emission, but a poor constraint on this parameter means that the grains could be larger or smaller, though by no more than an order of magnitude. We return to the likely size of blowout grains when considering small-grain dynamics in Section 4.2.

Figure 7 shows the star-subtracted SED and the disk model, and here the relative contributions of the two components as a function of wavelength can again be seen. We note that the ALMA fluxes $(1300 \mu \mathrm{m})$ lie somewhat above the fit, but are consistent with the model at the $2-\sigma$ level. We discuss the spectral index of the disk in Section 4.3 below. As noted above, at $70 \mu \mathrm{m}$ the emission from the planetesimal belt and halo components is similar, but at longer wavelengths the planetesimal belt dominates. The planetesimal belt is dominated by emission at $40 \mathrm{AU}$, where the grains have a temperature of $39 \mathrm{~K}$. The temperature of the halo component at that separation from the star is slightly higher $(50 \mathrm{~K})$, but poorly constrained due to the lack of resolution and degeneracy with $\lambda_{0}$ and $\beta_{\lambda}$. The fact that these two components yield a composite spectrum that is a simple blackbody is a reminder of the power of resolved imaging, since much information can be hidden within a single component SED.

\subsection{Central Asteroid Belt?}

MacGregor et al. (2013) found evidence of an excess above the photosphere at the position of the star in their high resolution ALMA imaging, and we include this component in the ALMA model image. The residuals from fitting our dust model to the Herschel and JCMT images in Figure 6 show no sign of any other unresolved excess above the stellar photosphere, so the best we can do is place an upper limit on the emission from such a centralized component of the pointsource sensitivity at a given wavelength (i.e., $3-\sigma$, MacGregor et al. 2013). It is also possible, given the considerably lower resolution of our observations, that such emission, if present, has been incorporated into the belt 


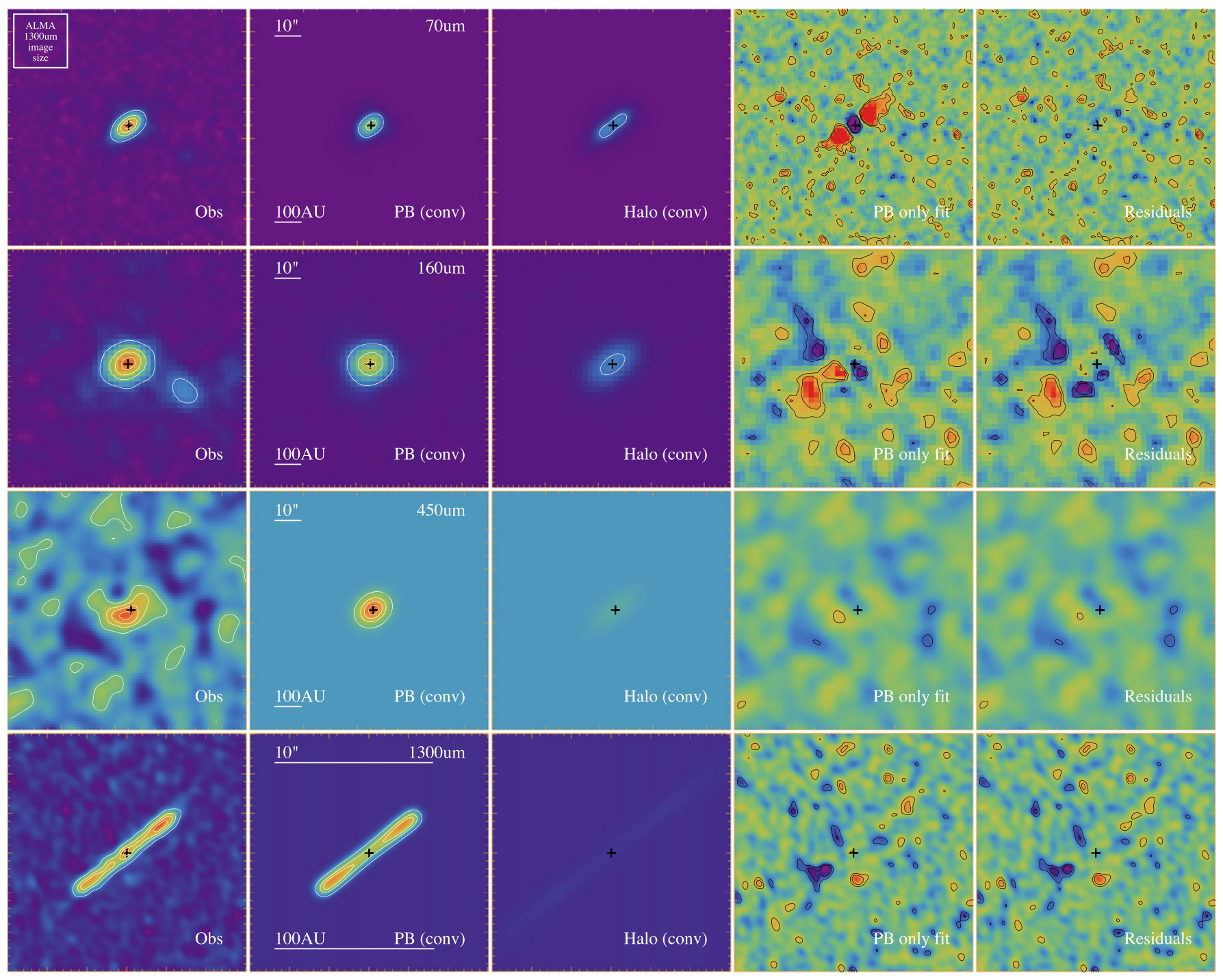

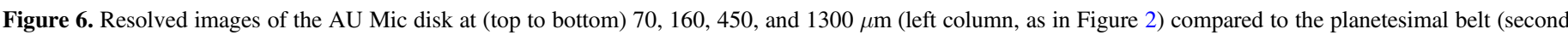

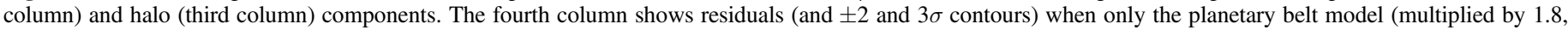

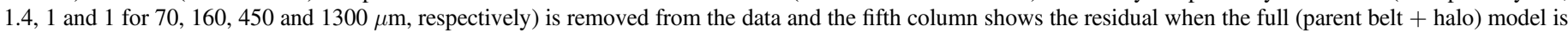

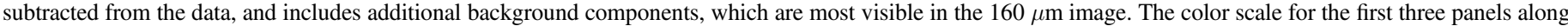

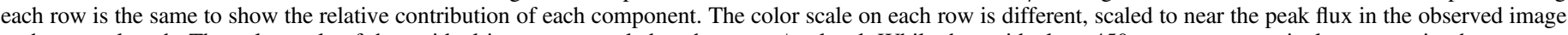

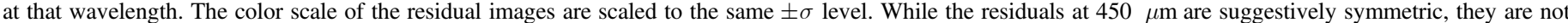

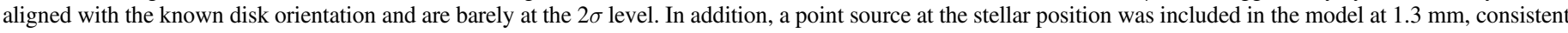

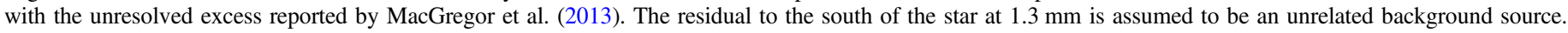

emission and removed, which adds uncertainty to the flux density of the belt.

From Figure 4 of Cranmer et al. (2013), the coronal model of AU Mic predicts a flux of a few $\mu \mathrm{Jy}$ around $100 \mu \mathrm{m}$ with a relatively flat distribution shortward of $1300 \mu \mathrm{m}$ where the ALMA detection was made. Our point source sensitivity is on the order of $1 \mathrm{mJy}$ at $70 \mu \mathrm{m}$ and $\sim 10 \mathrm{mJy}$ at $160 \mu \mathrm{m}$, meaning that, even in the absence of the disk, we would not be able to detect a coronal thermal heating contribution to the total flux in the Herschel data.

\subsection{Mass of the Disk}

A direct estimate of the mass of dust in AU Mic's debris disk can be made from its submillimeter flux densities. Debris disks are optically thin at these wavelengths, and so the mass of the disk is directly proportional to the emission, following the relation:

$$
M_{d}=\frac{F_{\nu} d^{2}}{\kappa_{\nu} B_{\nu}\left(T_{d}\right)}
$$

In the Rayleigh-Jeans limit, Equation (1) reduces to

$$
M_{d}\left[M_{\oplus}\right]=5.8 \times 10^{-10} \frac{F_{\nu}[\mathrm{mJy}](d[\mathrm{pc}])^{2}(\lambda[\mu \mathrm{m}])^{2}}{\kappa_{\nu}\left[\mathrm{cm}^{2} \mathrm{~g}^{-1}\right] T_{d}[\mathrm{~K}]} .
$$

While the AU Mic SED supports a single temperature fit of $53 \mathrm{~K}$, our image modeling requires a separate planetesimal belt of $39 \mathrm{~K}$ and a warmer halo, which contributes negligible flux density to the total emission at $850 \mu \mathrm{m}$ (see Figure 7). This temperature is comparable to the $40 \mathrm{~K}$ adopted by Liu et al. (2004) in the absence of an SED temperature fit. The color corrected flux density measured with SCUBA-2 at 


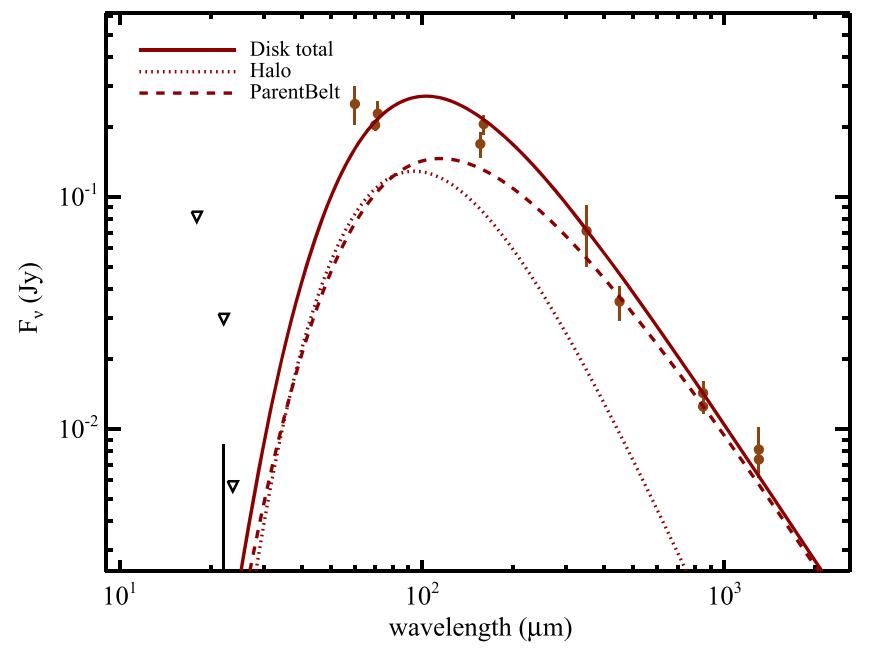

Figure 7. Flux distribution of the disk around AU Mic. Subtraction of the stellar fit from Figure 5 reveals the distribution of the disk itself. Mid-infrared upper limits are shown from WISE $(22 \mu \mathrm{m})$, Spitzer $(24 \mu \mathrm{m})$, and IRAS $(25 \mu \mathrm{m})$. Far-infrared detections at $60 \mu \mathrm{m}($ IRAS) and 70 and $160 \mu \mathrm{m}$ (Spitzer and Herschel) are shown. Submillimeter detections are from the CSO $(350 \mu \mathrm{m})$, JCMT $(450$ and $850 \mu \mathrm{m})$, and ALMA $(1.3 \mathrm{~mm})$. The model components of the planetesimal belt ("parent belt") and the halo are shown.

$850 \mu \mathrm{m}(12.5 \mathrm{mJy})$ is within the $1-\sigma$ limit of the value measured by Liu et al. (2004) with SCUBA $(14.4 \pm 1.8 \mathrm{mJy})$, although the SCUBA measurement relied on the single bolometer "photometry" mode, rather than a mapping technique, which could have missed some emission.

Taking $12.5 \mathrm{mJy}$ as the $850 \mu \mathrm{m}$ flux density of the disk yields a slightly lower $1 \mathrm{~mm}$ grain dust mass of $0.01 M_{\oplus}$ relative to the Liu et al. (2004) measurement, for the same dust opacity of $1.7 \mathrm{~cm}^{2} \mathrm{~g}^{-1}$. The dominant sources of uncertainty in the mass are the flux density and the temperature of the planetesimal component of the disk, amounting to a $20 \%$ uncertainty in the disk mass.

\section{DISCUSSION}

We now briefly consider the dust properties in more detail, using realistic grain models to compare the probable dust temperatures and sizes with those derived from the modeling.

\subsection{Temperature of AU Mic Dust Grains}

The SED fit to the disk around AU Mic requires only a single temperature component. The emission is well fit $\left(\chi^{2}\right.$ is minimized) from mid-IR through submillimeter wavelengths by a pure blackbody with a temperature of $53 \pm 2 \mathrm{~K}$. Figure 7 shows, however, that the halo and planetesimal belt components could still be segregated in temperature, with the parent bodies of the belt colder $(\sim 39 \mathrm{~K})$ than the halo (see also Fitzgerald et al. 2007).

Figure 8 shows the distribution of temperature with grain size for three grain compositions at the location of the planetesimal belt. The temperature distributions were calculated as in Augereau et al. (1999) and Wyatt \& Dent (2002). We used three different compositions: (1) a mix of one part amorphous astronomical silicates to two parts organics, (2) pure astronomical silicates, and (3) pure ice (Li \& Greenberg 1997). The models shown in Figure 8 are not porous. Porous grains do not have the peak in temperature near $1 \mu \mathrm{m}$, instead decreasing steadily to $20-25 \mathrm{~K}$ from maximum

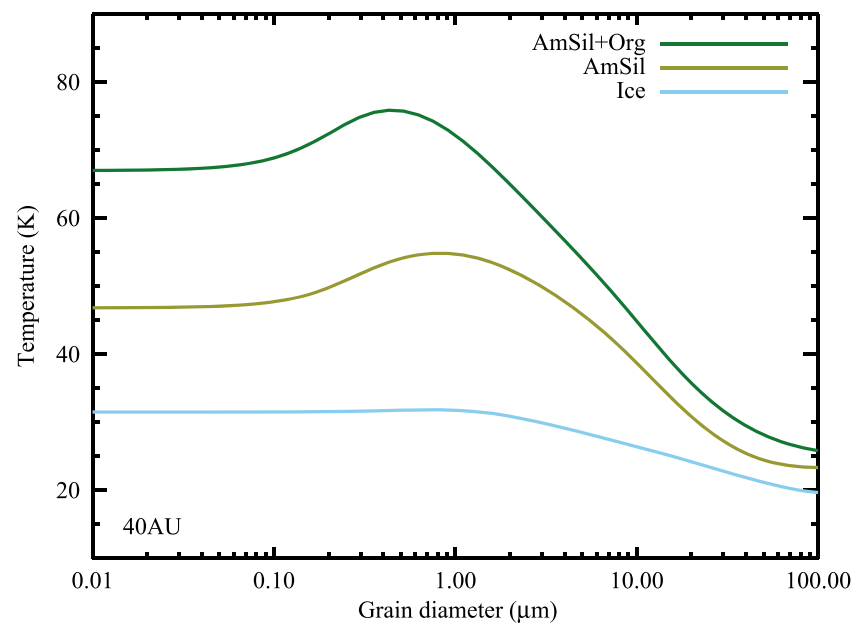

Figure 8. Temperature of grains as a function of diameter for three dust grain compositions. For the planetesimal belt temperature of $39 \mathrm{~K}$, pure icy grains are ruled out for all considered grain sizes for compact, spherical grains, though other compositional mixes including ices could potentially satisfy the modeled temperature. See the text for an explanation of the grain compositions.

temperatures similar to the non-porous case. It is clear that pure water ice grains are ruled out at $39 \mathrm{~K}$ for all grain sizes, although we note that other compositional mixes could be modeled to satisfy the temperature distribution we have derived.

\subsection{Small-grain Dynamics}

Orbiting dust is subject to forces from both radiation and stellar winds. Around early-type stars, radiation dominates, while stellar winds are thought to be more important for latetype stars such as AU Mic. These forces are commonly split into radial and tangential components. The radial component is called "radiation pressure" or the "radiation force," and effectively reduces the stellar mass seen by the particle, with an analogous effect for the stellar wind. The tangential component is called Poynting-Robertson drag, and causes dust grains to spiral into the star, again with an analogous (and much stronger) effect for stellar wind. See Burns et al. (1979) for a detailed review.

The small grain halo seen previously in scattered light and confirmed here in the far-IR is similar in morphology to those seen around other debris disks (e.g., $\beta$ Pic: Pantin et al. 1997; Augereau et al. 2001; HR 8799: Su et al. 2009; Matthews et al. 2014a). In other systems, the halo is attributed to the effect of the radial component of the radiation force from the star, which increases the eccentricity of smallest bound grains. The key measure of this effect is the parameter $\beta$, the ratio of the radiation force to the gravitational attraction of the star (distinct from the millimeter-wave spectral slope $\beta_{\lambda}$ used above). Grains with $\beta>1$ are unbound, and grains liberated from a parent body on a circular orbit are unbound if $\beta>0.5$. Figure 9 shows $\beta$ as a function of grain diameter, calculated for amorphous silicates + organics, and ice as described above. The solid curves show $\beta$ for the effect of radiation force on solid grains (left panel) and porous grains (right panel). It is clear that a range of $\beta$ is possible due to the poorly constrained grain compositions. Also, the assumption of Strubbe \& Chiang (2006) that the radiation force blowout grain size exists (i.e., that $\beta>0.5$ for any size) is not well founded, particularly if the grains are porous. Essentially, the low luminosity of an M-type 

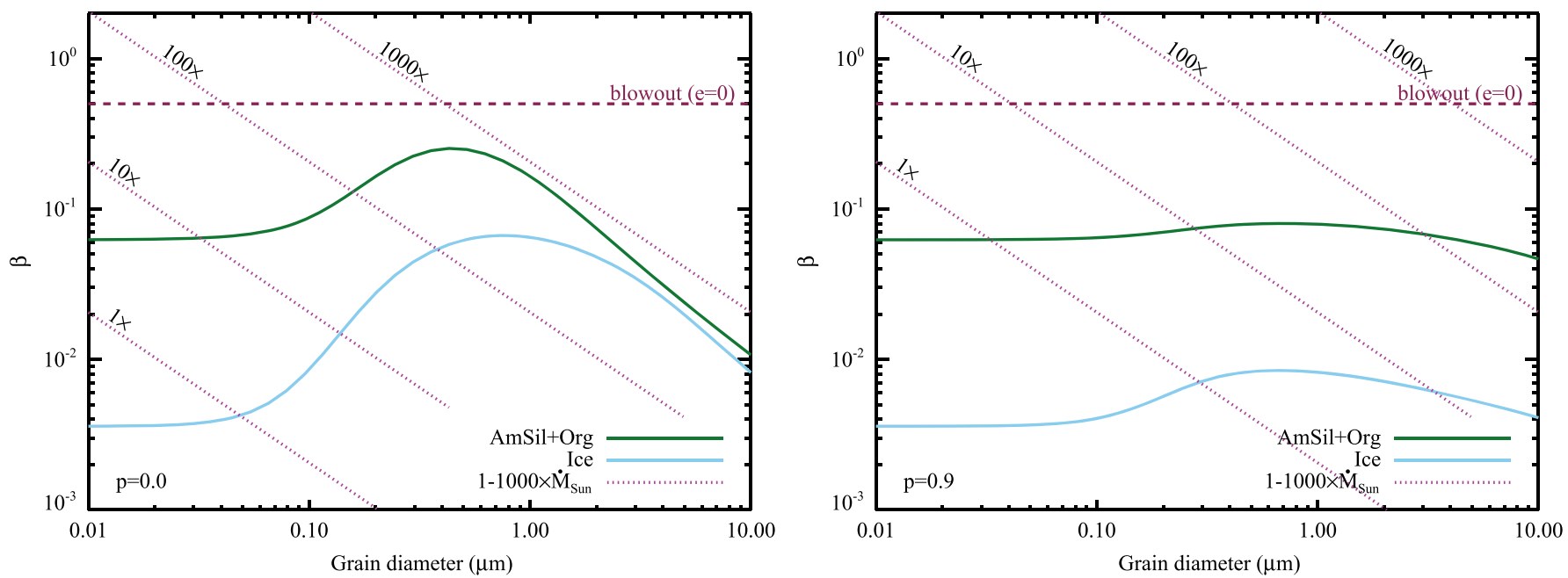

Figure 9. Effects of porosity. The ratio of radiative to gravitational forces $(\beta)$ as a function of grain composition is shown. For zero porosity grains, there is no grain size that reaches the blowout condition $(\beta=0.5)$ around $\mathrm{AU}$ Mic, although non-zero porosity can either decrease or enhance the $\beta$ ratio, depending on grain size. For the higher porosity grains, the $\beta$ values ultimately converge to be similar, following an inverse relation to grain size. Stellar wind effects on $\beta_{\mathrm{SW}}$ for $1,10,10^{2}$, and $10^{3} \times \dot{M}_{\odot}$ are shown as dotted lines. Empirical evidence for other stars suggests that AU Mic may have a stellar wind level as high as, but not much higher than, $10 \times \dot{M}_{\odot}($ Strubbe \& Chiang 2006).

star does not produce a strong enough radiation force to significantly affect the small grains. Even taking into account the flaring of the star is not enough to remove grains via the radiation force (Augereau \& Beust 2006).

Unlike early-type stars, an $\mathrm{M}$ star can have a relatively strong stellar wind (Plavchan et al. 2005). The force from this particle wind causes an effect similar to that of the radiation force and is able to reproduce the scattered light halo around AU Mic (Augereau \& Beust 2006). The dotted lines in Figure 9 demonstrate the effective $\beta_{\mathrm{SW}}$ parameter for the stellar wind force (using the prescription of Strubbe \& Chiang 2006). The left panel shows that for compact grains the wind blowout size is very small, $<0.1 \mu \mathrm{m}$ for the favored AU Mic mass-loss rate of $10 \times \dot{M}_{\odot}$ (Cully et al. 1994, based on EUV flaring). Such small grains are extremely poor scatterers of light, even at optical wavelengths, so they would appear very faint. Augereau \& Beust (2006) get around this problem by including flares, which increase the time-averaged mass-loss rate to $300 \times \dot{M}_{\odot}$. Such a high mass-loss rate would, however, make the system transport-dominated rather than collision-dominated, i.e., stellar wind drag would also become important, filling the region interior to $30 \mathrm{AU}$ with small dust grains in a manner contrary to the scattered light observations (Strubbe \& Chiang 2006). Lim \& White (1996) also point out that radio flares would be obscured if the stellar winds of M stars were many orders of magnitude more massive than the Sun's.

The right panel of Figure 9 shows that porosity can also play an important role. By increasing the grain porosity, the effect of wind force increases, in turn increasing the blowout grain size. For $p=0.9$, the blowout size is an order of magnitude larger for the same mass-loss rate, and the scattering efficiency of the grains greater, thus providing the likely solution. For a stellar wind rate of $\sim 100 \dot{M}_{\odot}$, the size of halo grains is roughly $0.1-1 \mu \mathrm{m}$. These grains have emission properties consistent with those derived from the image modeling above. This conclusion of high porosity compares well with Graham et al. (2007), Fitzgerald et al. (2007), and Shen et al. (2009) who all find that porosity is necessary to explain the scattering and polarization properties of the grains, although Shen et al.
(2009) find that a composition of random aggregates require a porosity of just $\sim 0.6$ compared to the $0.9-0.94$ required by Graham et al. (2007) for the Mie theory applied to spherical grains or aggregates.

The Strubbe \& Chiang (2006) model of the disk as a collision-dominated, narrow birth ring from which the smallest grains are blown out does a good job of explaining the halo and lack of small grains interior to $30 \mathrm{AU}$. The recent ALMA results of MacGregor et al. (2013), however, show that there are indeed larger grains interior to the presumed birth ring. Given the $1300 \mu \mathrm{m}$ observing wavelength, those observations should be dominated by grains with $a \sim \lambda / 2 \pi$, or $200 \mu \mathrm{m}$. Drag forces, which can act on larger grains over long timescales, are therefore a likely cause of this interior emission. Augereau \& Beust (2006) concluded that Poynting-Robertson drag is negligible for AU Mic and that stellar wind drag may evacuate the inner regions (see also Strubbe \& Chiang 2006). However, more recent work suggests that stellar wind drag can fill the interior regions to some degree, without seriously violating the scattered light constraints (Schüppler et al. 2015). Alternatively, the radially increasing surface density of this disk is reminiscent of a "self-stirred" disk that collisionally grinds down from the inside out (Kenyon \& Bromley 2002; Kennedy \& Wyatt 2010). Schüppler et al. (2015) also find that the self-stirred model is plausible for AU Mic's disk.

\subsection{Spectral Index at Millimeter Wavelengths}

The data compiled in this work provide a long lever arm to measure the spectral index, $\alpha$ where $F_{\nu} \propto \lambda^{-\alpha}$ is the emission from submillimeter through millimeter wavelengths. As noted above, the $1300 \mu \mathrm{m}$ fluxes from the SMA and ALMA both lie above the nominal "best fit" line of the models produced by fits to the SED and the images. Comparisons of the 350/1300 spectral index and the 450/1300 spectral index yields $\alpha$ values ranging from $\sim 1.5$ to close to 2.0 , which was the inferred spectral index of 350 versus 1300 (SMA) by Wilner et al. (2012). It is clear from the SED that these wavelengths are in the Rayleigh-Jeans part of the spectrum, which was not definite 
based on the data available to Wilner et al. (2012) and Gáspár et al. (2012).

A spectral index of $\sim 2$ is lower than most other debris disks. Based on the compiled data of Gáspár et al. (2012), the three A stars in that study ( $\beta$ Pictoris, Vega, and Fomalhaut) have the highest value of $\alpha$, which could suggest that the size distribution is different around A stars, being more consistent with a collisional cascade. Gáspár et al. (2012) show that for a collisional quasi steady-state at a single temperature, the Rayleigh-Jeans tail of the SED should yield a spectral index of 2.65 , for a self-similar collisional differential size distribution index of 3.65. The shallower slopes measured by Wilner et al. (2012) and a comparison of the $450 \mu \mathrm{m}$ flux density from SCUBA-2 of Table 2 (which actually seems to lie somewhat below the SED fit) and the elevated flux densities measured by the SMA and ALMA at $1300 \mu \mathrm{m}$ suggest a size distribution index of 3.0, i.e., that the size distribution is not the product of a collisional cascade. The shallow slope may however arise from the way that the parent belt and halo components add to yield the total spectrum (Figure 7, Fitzgerald et al. 2007). Such a shallow distribution constraint has also been noted for two IR bright disks (HD 377 and HD 104680) not detected with the VLA at $9 \mathrm{~mm}$ (Greaves et al. 2012). In AU Mic, it appears the grains may be very large $(>>1 \mathrm{~mm})$. Longer wavelength observations of the disk beyond $1300 \mu \mathrm{m}$ would be very beneficial to further constrain the size distribution.

The spectral slope is also dependent on composition. Schüppler et al. (2015) present more detailed modeling of the AU Mic disk, including investigation of the impact of compositional dependence on the derived spectral slope. They find a best fit for a combination of silicate, carbon, and vacuum in equal measure, with little change in the spectral slope fit with small additions of ice or variations of these materials. Their test also provides clear evidence for porous grains. As in our analysis, their SED fits also underestimate the $1300 \mu \mathrm{m}$ flux densities from SMA and ALMA, though, as in our case, this result should be interpreted with caution since the exploration of the parameter space is limited.

\section{CONCLUSIONS}

AU Mic's halo has been known since the earliest scattered light images of the disk (Kalas et al. 2004), which revealed a radial extent of $210 \mathrm{AU}$. ALMA imaging has confirmed a planetesimal belt at $\sim 40 \mathrm{AU}$, as predicted by Strubbe \& Chiang (2006) and Augereau \& Beust (2006) and consistent with a break in the surface brightness profiles observed in scattered light (Metchev et al. 2005; Fitzgerald et al. 2007; Graham et al. 2007). Using Herschel and SCUBA-2 at the JCMT, we have detected and modeled the thermal emission from both the planetesimal belt and the halo at wavelengths of 70, 160, 450, and $1300 \mu \mathrm{m}$, the latter being ALMA data as presented by MacGregor et al. (2013).

We present a simple spatial model that utilizes the existing model of the planetesimal belt from ALMA imaging to reconcile the ostensibly single-temperature blackbody SED of AU Mic's disk with the presence of emission from the extended halo. The best-fit model is consistent with the birth ring model explored in Wilner et al. (2012) and MacGregor et al. (2013), a planetesimal belt extending from 8.8 to $40 \mathrm{AU}$, but with the addition of a shallow surface density profile halo dominated by grains roughly $1 \mu \mathrm{m}$ in size.
We observe no asymmetries in the disk images, and the residual images all show that there is negligible emission unaccounted for by a smooth disk.

We confirm that AU Mic does not exert enough radiation force to blow out grains. We also find that for the inferred stellar mass-loss rate of 10 times solar, compact (porosity $=0$ ) grains can only be removed if they are very small; if the porosity reaches 0.9 or higher, then grains approaching $0.1 \mu \mathrm{m}$ can be removed. This result suggests that a higher mass loss is favored to place larger $\sim 1 \mu \mathrm{m}$ grains in the halo, and a high degree of porosity in the grains of AU Mic, consistent with previous work on the scattering and polarization properties of the disk at optical wavelengths.

The spectral index of the planetesimal belt of AU Mic may be more shallow than our modeling suggests, if the $450 \mu$ m diminished flux density and $1300 \mu$ m elevated flux densities are real. The spectral index established from 350 and $850 \mu$ m data was already shallow at a value of $\sim 2$, but may be as low as 1.5 , suggesting that the disk may have a grain size distribution inconsistent with that expected of a quasi steadystate collisional cascade.

We acknowledge the efforts of our referee, whose constructive comments improved and clarified the manuscript. B.C.M. is grateful to Christine Chen for checking the quality of high-res IRS data from Spitzer. G.M.K. acknowledges support from the European Union through ERC grant number 279973. M.B. acknowledges support from a FONDECYT Postdoctral Fellowship, project no. 3140479. P.K. thanks support from NASA NMO711043, NSF AST-0909188, and GO-10228 from STScI under NASA contract NAS5-26555. E.P. is partly supported by a AAS Chretien grant. PACS has been developed by a consortium of institutes led by MPE (Germany) and including UVIE (Austria); KU Leuven, CSL, IMEC (Belgium); CEA, LAM (France); MPIA (Germany); INAF-IFSI/OAA/ OAP/OAT, LENS, SISSA (Italy); IAC (Spain). This development has been supported by the funding agencies BMVIT (Austria), ESA-PRODEX (Belgium), CEA/CNES (France), DLR (Germany), ASI/INAF (Italy), and CICYT/MCYT (Spain). SPIRE has been developed by a consortium of institutes led by Cardiff University (UK) and including Univ. Lethbridge (Canada); NAOC (China); CEA, LAM (France); IFSI, Univ. Padua (Italy); IAC (Spain); Stockholm Observatory (Sweden); Imperial College London, RAL, UCL-MSSL, UKATC, Univ. Sussex (UK); and Caltech, JPL, NHSC, Univ. Colorado (USA). This development has been supported by national funding agencies: CSA (Canada); NAOC (China); CEA, CNES, CNRS (France); ASI (Italy); MCINN (Spain); SNSB (Sweden); STFC, UKSA (UK); and NASA (USA). This research used the facilities of the Canadian Astronomy Data Centre operated by the National Research Council of Canada with the support of the Canadian Space Agency.

\section{REFERENCES}

Allard, F., Homeier, D., Freytag, B., \& Sharp, C. M. 2012, in EAS Publications Ser. 57, Low-mass Stars and the Transition Stars/Brown Dwarfs EES2011, ed. C. Reylé, C. Charbonnel \& M. Schultheis, 3

Anglada-Escudé, G., \& Tuomi, M. 2012, A\&A, 548, A58

Augereau, J., \& Beust, H. 2006, A\&A, 455, 987

Augereau, J. C., Lagrange, A. M., Mouillet, D., Papaloizou, J. C. B., \& Grorod, P. A. 1999, A\&A, 348, 557

Augereau, J.-C., Nelson, R. P., Lagrange, A. M., Papaloizou, J. C. B., \& Mouillet, D. 2001, A\&A, 370, 447 
Balog, Z., Müller, T., Nielbock, M., et al. 2014, ExA, 37, 129

Binks, A. S., \& Jeffries, R. D. 2014, MNRAS, 438, L11

Booth, M., Kennedy, G., Sibthorpe, B., et al. 2013, MNRAS, 428, 1263

Burns, J. A., Lamy, P. L., \& Soter, S. 1979, Icar, 40, 1

Cavanagh, B., Jenness, T., Economou, F., \& Currie, M. J. 2008, AN, 329, 295

Chambers, J. E. 2014, Icar, 233, 83

Chapin, E. L., Berry, D. S., Gibb, A. G., et al. 2013, MNRAS, 430, 2545

Chen, C. H., Mamajek, E. E., Bitner, M. A., et al. 2011, ApJ, 738, 122

Chen, C. H., Patten, B. M., Werner, M. W., et al. 2005, ApJ, 634, 1372

Chen, C. H., Pecaut, M., Mamajek, E. E., Su, K. Y. L., \& Bitner, M. 2012, ApJ, 756, 133

Cranmer, S. R., Wilner, D. J., \& MacGregor, M. A. 2013, ApJ, 772, 149

Cully, S. L., Fisher, G. H., Abbott, M. J., \& Siegmund, O. H. W. 1994, ApJ, 435, 449

Dempsey, J. T., Friberg, P., Jenness, T., et al. 2013, MNRAS, 430, 2534

Dent, W. R. F., Wyatt, M. C., Roberge, A., et al. 2014, Sci, 343, 1490

Eiroa, C., Marshall, J. P., Mora, A., et al. 2013, A\&A, 555, A11

Fitzgerald, M. P., Kalas, P. G., Duchêne, G., Pinte, C., \& Graham, J. R. 2007, ApJ, 670, 536

Forbrich, J., Lada, C. J., Muench, A. A., \& Teixeira, P. S. 2008, ApJ, 687, 1107

Gáspár, A., Psaltis, D., Rieke, G. H., \& Özel, F. 2012, ApJ, 754, 74

Gautier, T. N., III, Rieke, G. H., Stansberry, J., et al. 2007, ApJ, 667, 527

Gorlova, N., Rieke, G. H., Muzerolle, J., et al. 2006, ApJ, 649, 1028

Graham, J. R., Kalas, P. G., \& Matthews, B. C. 2007, ApJ, 654, 595

Greaves, J. S., Hales, A. S., Mason, B. S., \& Matthews, B. C. 2012, MNRAS, 423, L70

Griffin, M. J., Abergel, A., Abreu, A., et al. 2010, A\&A, 518, L3

Holland, W. S., Bintley, D., Chapin, E. L., et al. 2013, MNRAS, 430, 2513

Holland, W. S., Greaves, J. S., Zuckerman, B., et al. 1998, Natur, 392, 788

Kalas, P., Liu, M. C., \& Matthews, B. C. 2004, Sci, 303, 1990

Kennedy, G. M., \& Wyatt, M. C. 2010, MNRAS, 405, 1253

Kennedy, G. M., Wyatt, M. C., Kalas, P., et al. 2014, MNRAS, 438, L96

Kennedy, G. M., Wyatt, M. C., Sibthorpe, B., et al. 2012a, MNRAS, 426, 2115

Kennedy, G. M., Wyatt, M. C., Sibthorpe, B., et al. 2012b, MNRAS, 421, 2264

Kenyon, S. J., \& Bromley, B. C. 2002, AJ, 123, 1757

Krist, J. E., Ardila, D. R., Golimowski, D. A., et al. 2005, AJ, 129, 1008

Lestrade, J.-F., Matthews, B. C., Sibthorpe, B., et al. 2012, A\&A, 548, A86

Lestrade, J.-F., Wyatt, M. C., Bertoldi, F., Dent, W. R. F., \& Menten, K. M. 2006, A\&A, 460, 733

Li, A., \& Greenberg, J. M. 1997, A\&A, 323, 566

Lim, J., \& White, S. M. 1996, ApJL, 462, L91

Liu, M. C. 2004, Sci, 305, 1442

Liu, M. C., Matthews, B. C., Williams, J. P., \& Kalas, P. G. 2004, ApJ, 608,526
Low, F. J., Smith, P. S., Werner, M., et al. 2005, ApJ, 631, 1170

MacGregor, M. A., Wilner, D. J., Rosenfeld, K. A., et al. 2013, ApJL, 762, L21

Malo, L., Doyon, R., Feiden, G. A., et al. 2014, ApJ, 792, 37

Mamajek, E. E., Bartlett, J. L., Seifahrt, A., et al. 2013, AJ, 146, 154

Mamajek, E. E., \& Bell, C. P. M. 2014, MNRAS, 445, 2169

Matthews, B., Kennedy, G., Sibthorpe, B., et al. 2014a, ApJ, 780, 97

Matthews, B. C., Kalas, P. G., \& Wyatt, M. C. 2007, ApJ, 663, 1103

Matthews, B. C., Krivov, A. V., Wyatt, M. C., Bryden, G., \& Eiroa, C. 2014b, Protostars and Planets VI, Vol. 521 (Tucson, AZ: Univ. Arizona Press)

Metchev, S. A., Eisner, J. A., Hillenbrand, L. A., \& Wolf, S. 2005, ApJ, 622,451

Moshir, M., Copan, G., Conrow, T., et al. 1990, in IRAS Faint Source Catalogue, version 2.0, 0

Ott, S. 2010, in ASP Conf. Ser. 434, Astronomical Data Analysis Software and Systems XIX, ed. Y. Mizumoto et al. (San Francisco, CA: ASP), 139

Pantin, E., Lagage, P. O., \& Artymowicz, P. 1997, A\&A, 327, 1123

Pecaut, M. J., \& Mamajek, E. E. 2013, ApJS, 208, 9

Phillips, N. M., Greaves, J. S., Dent, W. R. F., et al. 2010, MNRAS, 403, 1089

Pilbratt, G. L., Riedinger, J. R., Passvogel, T., et al. 2010, A\&A, 518, L1

Plavchan, P., Jura, M., \& Lipscy, S. J. 2005, ApJ, 631, 1161

Plavchan, P., Werner, M. W., Chen, C. H., et al. 2009, ApJ, 698, 1068

Poglitsch, A., Waelkens, C., Geis, N., et al. 2010, A\&A, 518, L2

Raymond, S. N., Armitage, P. J., Moro-Martín, A., et al. 2012, A\&A, 541, A11

Rebull, L. M., Stapelfeldt, K. R., Werner, M. W., et al. 2008, ApJ, 681, 1484

Rivera, E. J., Lissauer, J. J., Butler, R. P., et al. 2005, ApJ, 634, 625

Schneider, G., Grady, C. A., Hines, D. C., et al. 2014, AJ, 148, 59

Schüppler, C., Löhne, T., Krivov, A. V., et al. 2015, arXiv:1506.04564

Shen, Y., Draine, B. T., \& Johnson, E. T. 2009, ApJ, 696, 2126

Sibthorpe, B., Vandenbussche, B., Greaves, J. S., et al. 2010, A\&A, 518, L130

Sierchio, J. M., Rieke, G. H., Su, K. Y. L., et al. 2010, ApJ, 712, 1421

Strubbe, L. E., \& Chiang, E. I. 2006, ApJ, 648, 652

Su, K. Y. L., Rieke, G. H., Stansberry, J. A., et al. 2006, ApJ, 653, 675

Su, K. Y. L., Rieke, G. H., Stapelfeldt, K. R., et al. 2009, ApJ, 705, 314

Thureau, N. D., Greaves, J. S., Matthews, B. C., et al. 2014, MNRAS, 445,2558

Udry, S., Bonfils, X., Delfosse, X., et al. 2007, A\&A, 469, L43

van Leeuwen, F. (ed.) 2007, Hipparcos, The New Reduction of The Raw Data (ASSL vol. 350; Berlin: Springer)

Williams, J. P., \& Andrews, S. M. 2006, ApJ, 653, 1480

Wilner, D. J., Andrews, S. M., MacGregor, M. A., \& Hughes, A. M. 2012, ApJL, 749, L27

Wyatt, M. C. 2008, ARAA, 46, 339

Wyatt, M. C., \& Dent, W. R. F. 2002, MNRAS, 334, 589

Zuckerman, B., Song, I., Bessell, M. S., \& Webb, R. A. 2001, ApJL, 562, L87 